\title{
A gestão da atenção às urgências e o protagonismo federal
}

\author{
M anagement of attention to emergency rooms and the federal role
}

Gisele0 'D wyer ${ }^{1}$

${ }^{1}$ Departamento de Administração e

Planejamento em Saúde, Escola Nacional de Saúde Pública Sergio Arouca, Fundação O swaldo Cruz. Av. Leopoldo Bulhões $1.480 / 727$, M anguinhos. 21041-210 Rio de Janeiro RJ. odwyer@ensp.fiocruz.br
Abstract Attention to the emergency care has been criticized, and since 2002 the Brazilian State has assumed the efforts to standardize the level of attention. It was proposed the analysis of documents and acts based on the Structuration Theory that considers the mobilization of allocative and authoritarian resources as dimensions of structure in interaction, which would justify the legitimacy exercised since the establishment of regulations. The National Emergency Plan (PNAU) had as guides: the federal funding, re gionalization, professional education, management by urgency committees, and the expansion of the network. It wasidentified the density of the documental proposals as the facilitator trend of the structural resource, innovative due to the regionalization and responsibilization proposals presented by the several actors and by the alleged centrality of the user. The financing of SUS, despiteits persistent state of embarrassment, had no coercive action on the technology investment. Under the current administration there was a vigorous expansion and structuring of the network, which was made by a strong inflow of federal funds. Themanagement by committees should be investigated and points out how fragile the management of labor is.

Key words Health politics, Attention to emergency care, $\mathrm{H}$ ealth management
Resumo 0 atendimento às urgências/emergências tem sido alvo de críticas e o Estado brasileiro, a partir de 2002, assume o esforço de normatizar esse nível de atenção. Este estudo objetivou analisar a política de urgência a partir dos documentos e portarias, baseando-se na teoria da estruturação (Giddens), que relaciona a mobilização de recursos alocativos e autoritários como dimensões da estrutura em interação, o que justificaria a legitimação exercida a partir da sanção das normas. A Política Nacional de U rgência (PNAU) teve como marcos o financiamento federal, a regionalização, a capacitação dos profissionais, a gestão por comitês deurgência ea expansão da rede. Identifiquei a densidade das propostas documentais como a vertente facilitadora do recurso estrutural, inovadora pel as propostas de regi onal ização e de responsabilização dos diversos atores e pela pretensa centralidade no usuário. 0 financiamento do SUS, apesar do seu persistente estado de constrangimento, não teve ação coerciva sobre 0 investimento tecnológico. $\mathrm{Na}$ atual gestão, houve uma vigorosa expansão e estruturação da rede, quesó foi possível por um forte aporte de recursos federais.

A gestão pelos comitês precisa ser investigada e aponta-se como fragilidade a gestão do trabalho. Palavras-chave Políticas de saúde, Atenção às urgências, Gestão em saúde 
Introdução -

0 contexto de atenção às urgências

No Brasil, na maioria dos estados e municípios, os serviços de emergência sofrem o maior impacto da desorganização do sistema, se tornando o alvo preferido para as críticas ao modelo nacional de assistência à saúde ${ }^{1}$. Um importante motivo de crítica tem sido a superlotação dessas emergências e seu impacto negativo para os pacientes, gerando problemas de difícil gerenciamento².

A superlotação nas emergências aumenta 0 risco de mortalidade para os casos atendidos com atraso e causa descontentamento para os demandantes, independente da gravidade do caso. Outra consequência é a flexibilização nos padrões de cuidado e da ética dos profissionais de saúde que atuam na urgência³.

Para a reorganização e regulação desses serviços no âmbito do SUS, foi proposta a Política Nacional de Atenção às Urgências ${ }^{4}$ (PNAU), sendo o Serviço deAtendimento M óvel deU rgência ${ }^{5}$ (SAMU) escolhido como primeira estratégia de implementação dessa política. O SAMU (baseado no modelo francês) é composto por uma Central de Regulação M édica deU rgências e pelo componente assistencial, que comporta as ambulâncias básicas e avançadas (para situações especiais de transporte, as ambulanchas e motolâncias ${ }^{6}$ ). A Central de Regulação ${ }^{7}$ deve receber pedidos de atendimento de urgência da população ou profissionais de saúde por meio do tele fone 192 e atendêlos no domicílio ou em qualquer espaço público. A mesma passa a representar uma porta de comunicação aberta ao público, através da qual os pedidos de socorro são recebidos, avaliados e estratificados de acordo com a gravidade. Assim, abrem-se perspectivas para a estruturação da relação entre vários serviços e a qualificação dos fluxos dos pacientes na rede de atenção às urgências. Sob essa ótica, 0 SU S ganha um observatório permanente de saúde, que tem a possibilidade de identificar os de terminantes e a natureza dos problemas, bem como replanejar, de forma dinâmica, a assistência às urgências e à saúde ${ }^{3}$.

Recentemente, Carret et al. ${ }^{8}$ revisaram os estudos sobre utilização inadequada de serviços de emergência. Esses autores reforçam que os serviços de emergência devem ser utilizados em circunstâncias específicas e que a utilização inadequada é prejudicial para os pacientes graves e para os não graves, porque esses últimos, ao ele gerem o hospital para seu atendimento, não têm garantido 0 atendimento de seguimento. Indi- cam que 0 acesso à atenção básica reduz 0 uso inapropriado de serviços de emergência, apenas se o paciente tiver rápido acesso ao atendimento de urgência no âmbito da atenção básica ${ }^{8}$. Também recentemente, Bittencourt eH ortal e realizaram uma revisão sistemática da literatura sobre tipos de intervenções propostas para solucionar a superlotação dos serviços de emergência ${ }^{9}$. Os resultados dessa revisão desmistificam as barreiras de acesso como efetivas para conter a superlotação. Segundo os autores, a superlotação revela o baixo desempenho do hospital, assim como da rede. Destacam os serviços de urgência/ emergência como representantes do desempenho dos serviços de saúde e a importância do comprometimento do profissional desses serviços para a solução do problema?.

Pode-se concluir que, sem a revisão de todo o sistema de saúde, a emergência continuará a ser usada inapropriadamente pelos pacientes como alternativa para 0 atendimento primário. Restrições de acesso têm sido propostas como solução ${ }^{10}$ e aconteceram também no Brasi| ${ }^{11}$, mas são inaceitáveisna nossa realidade, em um país com tanta desigual dade social, além de não serem efetivas 9 .

Puccini eCornetta ${ }^{12}$, em um estudo demonitoramento em pronto-socorros de eventos sentinela da atenção básica, concluem que atender prontamente, ecom médico, éfundamental para a atenção básica e que é preciso superar sua deficiência numérica, organização fragilizada erecursos capengas em unidades "embaralhadas com as pretensas verdades nacionais do PSF"12.

Em função do exposto, é identificada a importância que a organização do sistema de urgência tem para a toda a rede, assim como o impacto dessa organização para a satisfação e 0 bom atendimento dos usuários.

Este texto pretendeu analisar os documentos que compõem a Política Nacional de Urgência e seu potencial para propiciar a integralidade do atendimento às urgências. $A$ análise foi baseada na teoria da estruturação de Giddens ${ }^{13}$, que considera a "estrutura" como o conjunto de regras e recursos implicados de modo recursivo, na reprodução social. Os recursos são as facilidades ou base de poder a que 0 agente tem acesso e que ele manipula para influenciar a interação com o outro. Os recursos autoritativos são capacidades que geram o comando sobre as pessoas (oportunidades na vida, posicionamento, organização e relação entre as pessoas). Os recursos alocativos são as capacidades sobre os objetos materiais ${ }^{13}$.

A partir da análise da PNAU, foram construídas as seguintes categorias: formulação de polí- 
ticas de saúde, protagonismo federal e alguns constrangimentos; contexto deformulação econsistência da PNAU; a gestão eos comitês gestores e expansão e estruturação da rede.

Formulação de políticas de saúde, protagonismo federal ealguns constrangimentos

Antes da análise das normas referentes à assistência às urgências, cabe uma brevereflexão sobre as disputas de poder envolvidas no processo deformulação e implantação das políticas em geral.

0 processo de formulação das políticas de saúde reflete disputas entre diferentes forças de poder, em que os formuladores, a partir de um problema ede uma intenção, propõem umanorma que reflete seus interesses, mas que foi produzida em um cenário de disputa ${ }^{14}$.

O SUS, enquanto política pública, constitui uma arena de conflitos, onde atuam diversos grupos deinteresse, queora contribuem para dar sustentação e legitimidade à política, ora estabelecem tensões para sua implementação ${ }^{15}$.

São dois os níveis de conflitos que mais interferem nesse processo político de formulação das normas e instituição do direito à saúde: 0 das fragmentações e dificuldades de relacionamentos entre os três poderes e entre os níveis de governo e os das disputas entre propostas concorrentes, com destaque para a convivência de um sistema público e de outro privado de assistência à saúde.

O Brasil caracteriza-se por um federalismo diferenciado pelas desigualdades sociais e regionais existentes, mas igualado pelos mecanismos dedescentralização, pactuação e participação que geram novas capacidades locais ${ }^{16}$. A centralização federal produz normas, incentivos financeiros e outros instrumentos de indução, enquanto a descentralização é apoiada por atores sociais e políticos das instâncias subnacionais ${ }^{17}$.

Portanto, as relações entre esferas de governo são complexas no Brasil. 0 atual arranjo federativo, ao definir simultaneamente as responsabilidades eos dispositivos de arrecadação nos diferentes níveis, disponibiliza incentivos para propiciar a adoção das políticas. Essa estrutura de incentivos é uma das estratégias para induzir os diversos atores a um comportamento cooperativo e é importante para estados e municípios, que são os agentes implementadores do SU S nos níveis regional elocal. Entretanto, a dependência do financiamento pode propiciar um cenário em que os entes federativos assumem um compor- tamento mais conflitivo pela disputa de recursos, do que cooperativo ${ }^{15}$.

Lima e Andrade ${ }^{18}$, com base no ano de 2005, constataram que as transferências federais de recursos orçamentários são fundamentais para os municípios com mais de cem mil habitantes. Além disso, destacam que as relações orçamentárias e fiscais estabelecidas no Brasil ainda são ineficazes na redução das desigualdades verificadas entre esses municípios ${ }^{18}$.

A partir dos Pactos da Saúde ${ }^{19}$, em 2006, o M inistério da Saúde tenta substituir a estratégia deinduzir decisões a partir deincentivos financeiros para estados e municípios pela estratégia da negociação permanente entre gestores centrada no compromisso político ${ }^{20}$. A estratégia indutora a partir de compromissos estabelecidos é mais coerente, só que, em cenários desubfinanciamento, a indução financeira tende a prevalecer.

Baptista ${ }^{21}$ diz que os instrumentos de indução política mais submetem os níveis subnacionais de governo às regras do sistema do quecompõem uma política acordada e sustentada nas diversas realidades institucionais do país e respaldas numa prática social concreta. Essa forma de atuar se reforça esemantém por uma postura também de acomodação das demais esferas de governo, que ainda percebem na relação de tutela com o M inistério da Saúde a garantia de benefícios e recursos para dar sustentação às suas políticas locais, sem uma responsabilização maior de seus orçamentos próprios ou o compromisso com uma agenda política mais extensiva ${ }^{21}$.

As "redes de políticas", conjuntos de atores que se articulam em uma área de interven ção a partir de objetivos particulares, seriam novas formas de intermediação de interesses, ora mais pluralistas, ora mais corporativistas, ao lado das práticas clientelistas e corporativistas tradicionais ${ }^{22}$. Trata-se de institucionalizar contrapesos ao poder via fortal ecimento dos mecanismos tradicionais de representação e/ou de novas formas de participação. A Rede Brasileira de Cooperação em Emergência (RBCE) cumpre esse papel de uma representação de interesses organizados na luta pela assi stência às urgências eéidentificada como um importante ator na formulação da PNAU. Essa rede surgiu na década de noventa a partir do interesse de médicos que atuavam na área. Ao longo dosanos, muitos outros profissionais se engajaram nessa rede, fundamental para a reflexão da atuação em urgências ${ }^{23}$.

Uma disputa com grande impacto para o direito à saúde é a coexistência de dois sistemas de saúde, o SUS e o sistema privado, consolidado 
nos anos noventa. Essa disputa está implicada com a imagem negativa do SUS. O poder executivo institui novas arenas de articulação de interesses no âmbito do subsistema privado ${ }^{24}$ que interferem nas bases materiais e políticas que redefinem o âmbito de atuação do SUS.

Fleury ${ }^{16}$ também aponta a crescente presença do mercado de seguros como um desafio para 0 SUS, que concorre com a ausência de recursos financeiros necessários ${ }^{16}$.

Esses desafios têm forte impacto sobre a incorporação dos profissionais de saúde no SUS. Segundo Machado et al. ${ }^{25}$, o SUS foi conduzido na década de noventa sob pressões para contenção de gastos com pessoal, em um contexto em que predominou uma agenda adversa à expansão do funcionalismo público e favorável à expansão de mecanismos de mercado, inclusiveno setor saúde. Os estados e municípios, na impossibilidade de contratação e remuneração adequadas de profissionais de saúde, muitas vezes recorreram às terceirizações e outras formas precárias de vinculação de profissionais no $\mathrm{SU} \mathrm{S}^{25} \mathrm{e}$ ainda recorrem.

Outra fragilidade para a gestão do trabalho em saúde e para as práticas dos profissionais éa desresponsabilização ${ }^{26}$ advinda da identificação de obstáculos estruturais e da falta de perspectivas de superação desses obstáculos. A disputa entre os sistemas público e privado tem servido para aprofundar essa "desresponsabilização", alimentando os constrangimentos para transformações e melhoria das práticas, além possibilitar práticas diferenciadas nos âmbitos privado e público ${ }^{27}$.

Com esses argumentos, pretendeu-se apontar a complexidade da relação entre estrutura e agente social, já que não existe uma estrutura que define tudo, muito menos agentes com autonomia plena ${ }^{13}$. Segundo Giddens, a constituição de agentes e estruturas não corresponde a dois conjuntos defenômenos dados independentemente - um dualismo -, mas representa uma dualidade ${ }^{13}$. Giddens faz uma reconciliação entre estrutura eação através da dualidade da estrutura, implicando agentes e recursos.

Portanto, a estrutura pode ser um elemento facilitador ou coercivo. Neste artigo, as políticas e seus documentos foram analisados como um recurso estrutural eum elemento facilitador para a gestão.

A pesar de todas as dificuldades apontadas, a formulação de propostas para orientar a implantação do SU Sem seus diversos aspectos exigeuma normatização dinâmica. Essedinamismo foi con- templado pela PNAU enquanto proposta para organizar 0 atendimento às urgências, o queserá defendido a seguir.

\section{Contexto deformulação econsistência da PNAU}

A criação da Coordenação Geral de Urgência e Emergência (CGUE), no âmbito do Ministério da Saúde, em 2003, foi fundamental para a implantação da política e gestão da atenção às urgências, apoiando sua efetivação.

0 documento que instituiu os Sistemas Estaduais deU rgência/Emergência, proposto em 2002 na gestão do M inistro Barjas N egri, foi norteador e precursor da PNAU ${ }^{28}$, contendo todos os elementos que foram desenvolvidos nas portarias seguintes. Entre eles, a integração dos níveis assistências na atenção às urgências; a regulação médica; a capacitação pelos Núcleos de Educação em Urgência (NEU) ea regionalização. Essa portaria ampliou a responsabilidade das ações de saúde no SUS, com responsabilização dos diversos profissionaise dos diferentes serviços, além de ampliar o horizonte de atuação, tornando mais resolutiva a atenção às urgências, além de permitir um diagnóstico das dificuldades da rede, possibilitando correções ${ }^{28}$.

Nesse momento, havia um consenso em relação à necessidade de intervenção nos serviços de saúde que sofriam constantes críticas da mídia. Grupos de interesse internos ao governo (Conselho Nacional de Saúde - CNS, Conselho Nacional de Secretários de Saúde - CONASS, Consel ho Nacional de Secretários Municipais de Saúde - CONASEM S e Comissão Intergestora Tripartite - CIT) assumiam essa discussão, que produziu diversas e legítimas contribuições, indicando uma participação ampliada no processo de formulação. Coube à gestão de Humberto Costa, o desenvolvimento dessas propostas, que puderam ser amadurecidas por um grupo ampliado de atores.

A RBCE atuou como grupo técnico e propositor. Esse grupo iniciou a discussão sobre esse nível deatenção com ampla participação de profissionais e gestores em $1995^{29}$.

A escolha do SAMU como primeiro componentea ser implantado foi estratégica, já que disponibilizava um novo serviço, propunha a regulação do sistema, a qualificação dos profissionais, além de ser observatório da rede. Entretanto, é destacado que na década de noventa os militares dos corpos de bombeiros já atuavam no 
atendimento pré-hospitalar móvel com base no modelo american ${ }^{29}$. O sformuladores da PNAU, entretanto, não valorizaram essa experiência. A falta de comunicação com Corporação dos Bombeiros gerou um problema de integração, como mostram Chomatas ${ }^{30}$, Deslandes et al..$^{31}$ e M inayo e Deslandes ${ }^{32}$.

Os documentos que compõem a PNAU são coerentes entre si e têm uma lógica de sequenciamento de propostas. Todos partem de uma consistente contextualização da atuação na área, destacando a baixa cobertura populacional ea insuficiente oferta de serviços de atendimento pré-hospitalar e a grande extensão territorial do país, com eventuais grandes distâncias para a rede hospitalar especializada e dealta complexidade, necessitando de serviços intermediários de complexidade ${ }^{28}$.

A Regulação M édica das Urgências ${ }^{29}$ é um documento produzido para a capacitação dos diferentes profissionais que atuam nas urgências. Nesse documento, éfeito destaque para o modelo assistencial ainda fortemente centrado na oferta de serviços e não nas necessidades dos cidadãos; a falta de acolhimento dos casos agudos de menor complexidade na atenção básica; a insuficiência de portas de entrada para os casos agudos demédia complexidade; a má utilização das portas de entrada da alta complexidade; a insuficiência de leitos hospitalares qualificados, especialmente de UTI e retaguarda para as urgências; as deficiências estruturais da rede assistencial áreas físicas, equipamentos e pessoal; a inadequação na estrutura curricular dos aparelhos formadores; o baixo investimento na qualificação e educação permanente dos profissionais de saúde; as dificuldades na formação das figuras regionais e fragilidade política nas pactuações; a incipiência nos mecanismos de referência e contrarreferência; as escassas ações de controlee avaliação das contratualizações externas e internase a falta de regulação 29 .

A partir desse cenário, são realizadas proposições visando à necessidade de definir uma ampla política nacional para a área, como sistemas regionalizados, com referências previamente pactuadas e efetivadas sob regulação médica, com hierarquia resolutiva e responsabilização sanitária, universalização do acesso, integralidade na atenção e equidade na alocação de recursos; a necessidade de integrar os serviços públicos e privados de atendimento pré-hospitalar móvel e de transporte inter-hospitalar à lógica dos sistemas de urgência, com regulação médica e equipe qualificada ea necessidade deestimular a criação de estruturas capazes de problematizar a reali- dade dos serviços e estabelecer o nexo entre trabalho e educação através dos $N$ úcleos de Educação em U rgências (NEU) ${ }^{28}$.

Ser "observatório de saúde e do sistema" é uma atribuição específica do SAM U em função da sua capacidade de monitorar, de forma dinâmica, sistematizada e em tempo real, todo seu funcionamento ${ }^{29}$. Ter essa incumbência reforça, como um dos produtos da regulação, a produção de informaç̧ões regulares para melhoria do sistema. Essa função exige um sistema de informação estruturado e é uma atividade primordial para a gestão da assistência.

A PNAU faz uma grande aposta no médico regulador e sua atuação para a organização do sistema. Esse profissional tem prerrogativas de autoridade para alocar pacientes dentro do sistema, comunicando sua decisão aos médicos assistentes das portas de urgência, a chamada"vaga zero" para internação. Esta é uma prerrogativa queprevêquea instituição, ao receber o paciente, não poderá recusá-lo al egando falta de vaga ${ }^{29}$.

A descrição das atribuiç̧ões deixa claro que a decisão de condução do caso é do regulador e indica a utilização do conceito ampliado de urgência. A gravação dos chamados dá respaldo a todos - solicitantes, regulador e receptor -, no sentido do registro correto e claro das informações, bem como da manutenção e observância de pactos e posturas éticas no trato dos pacientes acometidos por urgência que, pela gravidade do caso, perdem o direito à escolha e são submetidos a tratamentos cuja autorização a sociedade delegou ao médico ${ }^{29}$.

Há uma proposta para al ocação de profissionais na regulação por critério populacional ${ }^{29}$. Também é proposto o quantitativo de ambulâncias por SAM U pelo mesmo critério populacional. É fundamental destacar quea PNAU propõe a assistência a partir de dois tipos de ambulância, a unidade de suporte básico (USB) e a unidade de suporte avançado (USA). Na básica, a equipe de saúde é composta apenas pelo técnico de enfermagem. $\mathrm{Na}$ avançada, trabalham $\mathrm{O}$ médico e o enfermeiro. No SAMU francês, que serviu de inspiração para o modelo brasileiro, só atuam ambulâncias com médico.

No Estado do Rio de Janeiro, a regulação nas três centrais dos SAM U sofreu distorções depráticas de alocação de ambulâncias ${ }^{23}$ que, além da insuficiência da frota, refletem a insuficiência da discussão dos usos de ambulâncias básicas e avançadas.

O SAMU, a Central de Regulação e o Núcleo de Educação deveriam ser implantados simulta- 
neamente, o queainda não acontece, como apontam autores que investigaram os núcleos de educação em urgên cias ${ }^{30,33,34}$ e a regulação ${ }^{23}$.

Após essa contextualização mais estrutural da rede, é importante resgatar a forma como esses documentos pensam o usuário e sua relação com o sistema. Destacam-se que o Sistema Estadual deUrgência eEmergência deveseestruturar a partir das necessidades sociais em saúde e sob o imperativo das necessidades humanas nas urgências; o diagnóstico destas necessidades deve ser feito a partir da observação e da avaliação dos territórios sociais com seus diferentes grupos e o confronto das necessidades com as ofertas permite visualizar as deficiências do sistemae projetar suas correções, sustentado por políticas públicas orientadas pela equidade ${ }^{28}$.

A pesar de haver o reconhecimento das necessidades não atendidas dos cidadãos em função deum modelo assistencial ainda fortementecentrado na oferta de serviços, não há aprofundamento na análise das necessidades da população. Há uma crítica ao modelo técnico-assistencial eà uma redefortemente influenciada por interesses de mercado que almeja o consumo de alta densidade tecnológica. A crítica embutida é da não valorização da resolutividade das ações dos serviços de menor densidadetecnológica, ou seja, atenção básica. Portanto, é feita uma legítima crítica sobre o consumo indevido de tecnologias, mas não há uma reflexão sobre estratégias para 0 atendimento das necessidades não atendidas da população. Ao transitar entre os legítimos e necessários critérios técnicos e 0 atendimento ampliado de necessidades, há um questionamento sobre os tradicionais conceitos de urgência eemergência.

Em função do grande número de dúvidas e ambivalência da terminologia, o documento opta por utilizar o termo "urgência" para todos os casos que necessitem de cuidados agudos, classificando o grau de urgência em níveis, tomando como marco ético de avaliação o "imperativo da necessidade humana $a^{29 " .}$

0 conceito ampliado de urgência difere de acordo com quem percebe ou sente. Para os usuários e familiares, pode estar associado a uma ruptura do curso da vida, que pode ser traduzida pela frase "eu não posso esperar". Para o médico, a urgência repousa não sobre a ruptura, mas sobre o tempo relacionado com o prognóstico vital em certo intervalo: "ele não pode esperar". Uma das atribuições da regulação éser mais permeável ao entendimento do paciente do queéurgência ${ }^{29}$.
0 documento propõe uma classificação de níveis de urgência que vai do 1 ao 4, sendo 01 prioridade absoluta (risco imediato de vida), 02 prioridade moderada (atendimento em poucas horas), 03 prioridade baixa (atendimento pode aguardar várias horas) e 04 prioridade mínima, cabendo nesse caso orientações por telefon $e^{29}$.

Há uma crítica aos serviços de pronto atendimento como porta de entrada para o sistema ${ }^{29}$. Entretanto, a urgência como "porta de entrada" responde a uma expectativa da população e essa porta de entrada "irregular" não compete com a atenção básica porquenão oferece condições necessárias para atuar como porta de entrada qualificada, como ser responsável pelo acompanhamento, seguimento e vínculo com o paciente. Cabeà atenção básica receber o paciente por demanda espontânea ou programada. Muito da demanda espontânea éatendimento deurgência, reconhecida ou não pelo referencial técnico. 0 utra grande parte da demanda espontânea não chega à atenção básica e a central de regulação permite um espaço de encaminhamento dessa demanda enquanto porta de entrada do sistema. Não seria a porta de entrada principal e desejada, mas uma porta de entrada possível eoperativa, já que encaminharia o paciente a partir dos recursos da rede. Em estudo sobre o SAMU no Estado do Rio deJ aneiro, essa atribuiç̧ão de "porta de entrada do sistema" foi bem evidenciada23.

\section{A gestão e os comitês gestores}

A definição das atribuições e responsabilidades dos gestores do SUS reconhece o papel fundamental dos municípios na execução da atenção pré hospitalar. D efine que cabeao estado a coordenação ea regulação geral do sistema ea operacionalização de suas ações de acordo com pactos estabelecidos, operando ativamenteno sentido da construção e ordenamento dos sistemas regionais ${ }^{28}$.

Como os critérios para alocação de ambulâncias (tipo e número) são definidos por base populacional, os recursos para equipamentos e área física também são proporcionais à base populacional. Os recursos federais podem ser transferidos para estados ou municípios, após aprovação do projeto de implantação de SAM U ou Unidade de Pronto Atendimento (UPA). As despesas de custeio são de responsabilidade compartilhada, sendo 50\% da União.

Além das atribuições gestoras de cada ente da federação, é prevista a formação de comitês ges- 
tores ${ }^{35}$ para os diversos níveis de atuação em urgências. Essa instância gestora, proposta em 2003, não havia sido apontada no documento precursor da política ${ }^{28}$.

A partir de então, há a indicação formal do espaço do comitê como avaliador do sistema e a necessidade de criação de comitês nos âmbitos estaduais, municipais, além dos regionais. Entre suas atribuições, destacam-se: articular os entes gestores e os executores das ações rel ativas à atenção às urgências; avaliar e pactuar as diretrizes e ações prioritárias na atenção às urgências, subordinadas às estruturas de articulação e gestão do SUS, nos seus vários níveis e anal isar sistematicamente os indicadores dos SAM U -192, buscando construir um quadro descritivo detalhado da atenção às urgências, para subsidiar ações intersetoriais ${ }^{35}$. Os comitês gestores têm grande importância para o planejamento do atendimento, especial mente os regionais pela dificuldade dessa nova forma de trabalho, integrar serviços a partir de interesses de vários e diferentes municípios.

Há proposição de indicadores de desempe nho a serem sistematicamente produzidos nos SAMU e analisados pelos respectivos comitês gestores. ${ }^{5}$. Alguns desses indicadores podem ser produzidos a partir do próprio sistema de informação do SAMU, que é disponibilizado para as centrais de regulação. São indicadores compatíveis com a expectativa deser observatório do SUS eque podem ser facilmente analisados e comparados por região. Infelizmente, há evidências de quenão estão sendo produzidos regularmente ${ }^{23}$.

\section{Expansão e estruturação da rede}

A estruturação da rede de aten ção às urgênciasfoi pensada no documento que foi um marco para a PNAU ${ }^{28}$. Essa primeira proposta pretendia dar conta da interiorização da assistência e da adequação dos pronto atendimentos, criticados por sua superlotação, pela priorização das urgências "sangrantes e ruidosas" e por cumprirem papel de escoamento das demandas reprimidas não satisfeitas na atenção primária com uma enorme produção de "consultas de urgência", ineficazes, paliativas e baseadas nos sintomas ${ }^{28}$.

A expansão dos SAMU foi expressiva e não seguiu nenhum padrão identificável. Em 2004, eram 27 SM AU e, em 2009, existiam 147 SAM U com cobertura de mais de 112 milhões de brasileiros (dados colhidosno sitedo M inistério da Saúde, 2009).
Atualmente, há a indicação de que os projetos autorizados sejam de SAM U regionalizado.

A partir de 2008, há novo incremento da expansão da rede pela proposta das U PA ${ }^{36}$. Com a UPA, tem-se a proposição de um novo espaço de aten ção, além da regionalização e qualificação da atenção, e da interiorização com ampliação do acesso, com vistas à equidade. Para favorecer a regionalização, éexigida a integração daU PA com SAMU e com o desenvolvimento da atenção básica, diferenciando-se dos tradicionais serviços de pronto atendimento ou pronto-socorros ${ }^{36}$.

Em 2009, são propostas novas diretrizes para a UPA ${ }^{37}$, que substituem as anteriores ${ }^{36}$. A maior precisão sobre financiamento para implantação das unidades e ampliação das fontes de recursos são as diferenças mais significativas entre as duas propostas.

Segundo o Secretário de Estado de Saúde e Defesa Civil, "as UPA no Rio de Janeiro foram implantadas como estratégia compensatória da insuficiência da atenção básica e da superlotação das emergências hospitalares no município do Rio de Janeiro ${ }^{38 " . ~ H a v i a ~ p r e v i s a ̃ o ~ d e ~ u m a ~ g r a n d e ~}$ expansão até 2010, que vem ocorrendo. Atéabril de2009, tinham sido inauguradas vinteU PA nesse município, que tem uma cobertura de PSF muito baixa se comparada com outras capitais com mais de um milhão de habitantes ${ }^{39}$. 0 destaque ao município do Rio contradiz o critério de interiorização, estando a justificativa da proposta no grande déficit de atenção básica.

Lindblom ${ }^{40}$ defendea existência de dois componentes no processo decisório político: a análise (medianteinformação, de preferência científica) e a política (mediante uso do poder). Esses componentes podem conflitar ou se complementar. Cabe questionar se a U PA tem sido proposta como necessidade técnica ou pelo seu uso político e se esses componentes conflitam ou se complementam. Como proposta de curto prazo, a U PA tem grandeimpacto político eleitoral egrande visibilidade. Entretanto, essa estratégia deve ser problematizada, inclusive sob o ponto de vista econômico.

Identifica-secomo outra estratégia de expansão da redeo Programa de Qualificação da Atenção Hospitalar de Urgência no Sistema Ú nico de Saúde- Programa QualiSUSUrgência ${ }^{41}$. Háuma intenção de, a partir de um programa já existente, o QualiSU ${ }^{42}$, que na sua origem já priorizava a assistência às urgências, resgatá-lo com um enfoque especial na área de urgências. 


\section{Conclusão}

A PNAU teve como marcos o financiamento federal ea regional ização. 0 financiamento do SUS, apesar do seu persistente estado de constrangimento, não teve ação coerciva sobre o investimento tecnológico para a área. 0 atual M inisté rio da Saúde priorizou implementar um vigoroso programa de investimento para alterar a configuração de Redes de Atenção à Saúdee a expansão da RedeN acional SAM U 192, queforam justificadas como "retaguarda adequadamente estruturada e qualificada" $36,37,41$. As UPA e motolâncias, instituídas na sua gestão, são estratégias de estruturação de curto prazo (UPA) e complementares (motolâncias), que só foram possíveis a partir de um forte aporte de recursos federais.

Uma fragilidade da política é a gestão do trabalho, na qual ainda não ocorreu 0 avanço ne cessário. N o Estado do Rio de Janeiro, não houve investimento no quesito qualificação, fixação e não precarização de profissionais ${ }^{23}$. A implantação dos SAMU eUPA tem ocorrido a partir de uma plasticidade na forma de contrato dos profissionais que não favorece a sustentabilidade da política, com maior impacto para o médico.

As dificuldades com a gestão de pessoal não são exclusivas do Rio de Janeiro ou do SAMU, UPA e emergência hospitalar. A própria expansão do PSF pretendida para o Rio de Janeiro cer- tamente sofrerá as mesmas restrições. Especialmente neste estado, a Corporação dos Bombeiros tem assumido parte da contratação dos profissionais do SAM U eUPA, enfrentando a precariedade vigente.

Os documentos que compõem a PNAU são coerentes, consistentes, dialogam entre si e projetam uma evolução da política. Ela é inovadora pelas propostas de regionalização e de responsabilização dos diversos atores e pela pretensa centralidade no usuário. A regionalização a partir da urgência é emblemática para a regional ização da assistência de forma mais abrangente.

A implementação da PNAU deve ser avaliada nos diversos estados e regiões. Pela pouca literatura existente sobre 0 atendimento pré-hospitalar de urgência, imagina-se que a consolidação de uma proposta nacional deva ter produzido experiências diferentes nos estados. Já foi anunciada essa especificidade ao mencionar o Rio deJ aneiro.

A regulação do sistema proposta a partir da urgência deve ser temporária. A atenção básica com um PSF mais estruturado, com equipe multiprofissional que realmente acompanhe os indivíduos, deve regular o sistema. Portanto, são muitos os desafios para a gestão das urgências em todos os níveis de governo e, na ausência de uma regulação eintegração entre os serviços mais efetiva, cabe a todos os níveis de atenção às urgências acolher as atuais demandas. 


\section{Referências}

1. Scarpelini S. A organização do atendimento às urgências e trauma. M edicina 2007; 40(3):315-320.

2. O 'Dwyer G, Oliveira S, De Setta M H. Avaliação dos serviços hospitalares de emergência do programa QualiSUS. Cien Saude Colet 2009; 14(5):1881-1890.

3. Santos JS, Scarpelini S, Brasileiro SLL, Ferraz, CA, Dallora MELV, Sá, MFS. Avaliação do modelo de organização da unidade de emergência do HCFMRP-USP, adotando, como referência, as políticas nacionais de atenção às urgências e de humanização. M edicina 2003; 36(2/4):498-515.

4. Brasil. M inistério da Saúde. Portaria no 1.863, de 29 de setembro de 2003. Institui a Política Nacional de Atenção às U rgências. Diário Oficial da U nião 2003; 06 out.

5. Brasil. Ministério da Saúde. Portaria no 1.864, de 29 de setembro de 2003. Institui o Componente préhospitalar móvel da PNAU - o SAMU. Diário Oficial da União 2003; 06 out.

6. Brasil. Ministério da Saúde. Portaria no 2.971, de 8 de dezembro de 2008. Institui o Veículo motocicleta - motolância. Diário Oficial da União 2008; 09 dez.

7. Brasil. M inistério da Saúde. Portaria no 2.657, de 16 de dezembro de 2004. Estabelece as atribuições das Centrais de Regulação M édica de U rgências. Diário Oficial da União 2004; 17 dez.

8. Carret MLV, Fassa AG, Domingues MR. Prevalência e fatores associados ao uso inadequado do serviço de emergência: uma revisão sistemática da literatura. Cad Saude Publica 2009; 25(1):7-28.

9. Bittencourt RJ, Hortale VA. Intervenções para solucionar a superlotação nos serviços de emergência hospitalar: uma revisão sistemática. Cad Saude Publica 2009; 25(7):1439-1454.

10. McGugan EA, Morrison W. Primary care or $A \& E$ ? A study of patients redirect from accident $\&$ emergency departments. Scott M ed J 2000; 45(5):144-147.

11. O 'D wyer G, Matta IEA, Pepe VLE. Avaliação dos serviços hospitalares de emergência do estado do Rio de Janeiro. Cien Saude Colet 2008; 13(5):16371648.

12. Puccini T, Cornetta VK. Ocorrências em prontosocorro: eventos sentinela para monitoramento da atenção básica de saúde. Cad Saude Publica 2008; 24(9):2032-2042.

13. Giddens A. A constituição da sociedade. São Paulo: M artins Fontes; 1984.

14. Walt G. Health Policy. An Introduction to Process and Power. Johannesburg: Witwatersrand University Press; 1994.

15. Viana ALD, Faria R, Pacífico H. O SUS e as novas práticas de avaliação para gestão em redes: é possível conciliar a visão clássica de avaliação como novas práticas? In: Pinheiro R, M attos RA, organizadores. Gestão em redes: práticas de avaliação, formação e participação na saúde. Rio de Janeiro: IM SUERJ/Abrasco; 2006. p. 135-150.

16. Fleury S. Reforma sanitária brasileira: dilemas entre 0 instituinte e 0 instituído. Cien Saude Colet 2009; 14(3):743-752.

17. Viana ALD, Machado CV. Descentralização e coordenação federativa: a experiência brasileira na saúde. Cien Saude Colet 2009; 14(3):807-817.
18. Lima LD, Andrade CLT. Condições de financiamento em saúde nos grandes municípios do Brasil. Cad Saude Publica 2009; 25(10):2237-2248.

19. Brasil. M inistério da Saúde. Portaria no 399, de 22 de fevereiro de 2006. Diretrizes do Pacto pela Saúde - Consolidação do Sistema Ú nico de Saúde. Diário 0 ficial da União 2006; $23 \mathrm{fev.}$

20. Paim JS, Teixeira CF. Configuração institucional e gestão do SUS: problemas e desafios. Cien Saude Colet 2007; 12(Supl):1819-1829.

21. Baptista TWF. Análise das portarias ministeriais da saúde e reflexões sobre a condução nacional da política de saúde. Cad Saude Publica 2007; 23(3):615626.

22. Castro M HG. Interesse, organizações e políticas sociais. BIB 1991; 31:17-48.

23. O 'Dwyer G. O princípio da integralidade e o SAM U [tese]. Rio de Janeiro (RJ): Instituto de Medicina Social, Universidade Estadual do Rio de Janeiro; 2009.

24. Bahia L. O sistema de saúde brasileiro entre normas e fatos: universalização mitigada e estratificação subsidiada. Cien Saude Colet 2009; 14(3):753-762.

25. Machado CV, Baptista TWF, Lima LD. Responsabilidade do Estado, papel e relações entre poderes na saúde: duas ou três coisas que sabemos disso. In: Pinheiro R, M attos RA, organizadores. Razões públicas para a integralidade em saúde: 0 cuidado como valor. Rio de Janeiro: IMS-UERJ/CEPESC/Abrasco; 2007. p. 42-62.

26. M attos RA. Integralidade, trabalho, saúde e formação profissional. In: Matta GC, Lima JCF, organizadores. Estado, sociedade e formação profissional em saúde: contradições e desafios em 20 anos de SUS. Rio de Janeiro: Fiocruz; 2008. p. 313-352.

27. Mattos RA. Princípios do Sistema Ú nico de Saúde (SUS) e a humanização das práticas de saúde. Interface (Botucatu) 2009; 13(supl.1):771-780.

28. Brasil. M inistério da Saúde. Portaria no 2.048, de 05 de novembro de 2002. Aprova o Regulamento Técnico dos Sistemas Estaduais de U rgências e Emergências. Diário Oficial da União 2002; 12 nov.

29. Brasil. Ministério da Saúde. Regulação M édica das U rgências: normas e $M$ anuais T écnicos do M inistério da Saúde. Brasília: M inistério da Saúde; 2006.

30. Chomatas $M$. Integração de saberes e práticas na organização do SAMU de Curitiba - PR. Divulg Saude Debate 2005; 32:90-95.

31. Deslandes SF, M inayo MCS, Souza ER, Carmo CN, Souza VR. Escolhas, Alianças e Vivências na Dinâmica da Pesquisa. In: M inayo MCS, Deslandes SF, organizadores. Análise diagnóstica da Política Nacional de Saúde para redução de acidentes e violências. Rio de Janeiro: Fiocruz; 2007. p. 31-46.

32. M inayo MCS, Deslandes SF. Análise da implantação do sistema de atendimento pré-hospitalar móvel em cinco capitais brasileiras. Cad Saude Publica 2008; 24(8):1877-1886.

33. Almeida AC. A avaliação da implantação e do desenvolvimento do sistema público municipal de atendimento pré hospitalar móvel da cidade do Recife [dissertação]. Recife (PE): Centro de Pesquisas Ageu M agalhães, Fiocruz; 2007. 
34. Assis SG, Deslandes SF, M inayo M CS. Prevenção, monitoramento, capacitação e pesquisa. In: Minayo M CS, Deslandes SF, organizadores. Análise diagnóstica da Política Nacional de Saúde para redução de acidentes e violências. Rio de Janeiro: Fiocruz; 2007. p.193-218.

35. Brasil M inistério da Saúde. Portaria № 2.072, de 30 de outubro de 2003. Institui o Comitê Gestor Nacional de Atenção às U rgências. Diário Oficial da U nião 2003; 31 out.

36. Brasil. M inistério da Saúde. Portaria no 2.922, de 2 de dezembro de 2008. Propõe a implantação/adequação de Unidades de Pronto Atendimento - UPA. Diário Oficial da União 2008; 03 dez.

37. Brasil. M inistério da Saúde. Portaria no 1.020, de 13 de maio de 2009. Estabelece diretrizes para a implantação do componente pré-hospitalar fixo (Unidades de Pronto Atendimento - UPA). Diário Oficial da União 2009; 15 mai.

38. Cortes S. Superlativo de acertos. 0 Globo 2009; 23 mar.

39. Souza MF, Hamann EM. Programa de Saúde da Família (PSF): uma agenda incompleta? Cien Saude Colet 2009; 14(supl. 1):1325-1339.

40. Lindblom CE. O processo de decisão política. (Cole ção Pensamento Político). Brasília: UNB; 1981.

41. Brasil. M inistério da Saúde. Portaria no 3.125, de 7 de dezembro de 2006. Institui o QualiSUS -U rgência. Diário Oficial da U nião 2006; 08 dez.

42. Brasil. M inistério da Saúde. Projeto de Investimento na Qualificação do SUS - QualiSUS. Brasília: Ministério da Saúde; 2004.

Artigo apresentado em 29/04/2010

Aprovado em 25/05/2010

Versão final apresentada em 31/05/2010 